\title{
Clinicians' attitudes and perceived barriers and facilitators to cancer treatment clinical practice guideline adherence: a systematic review of qualitative and quantitative literature
}

Mia Bierbaum ${ }^{*}$, Frances Rapport ${ }^{1}$, Gaston Arnolda ${ }^{1,2}$, Brona Nic Giolla Easpaig ${ }^{1,2}$, Klay Lamprell ${ }^{1,2}$, Karen Hutchinson ${ }^{1}$, Geoff P. Delaney ${ }^{2,3,4,5}$, Winston Liauw ${ }^{2,4,6}$, Richard Kefford ${ }^{2,7}$, lan Olver ${ }^{2,8}$ and Jeffrey Braithwaite ${ }^{1,2}$

\begin{abstract}
Background: Clinical Practice Guidelines (CPGs) synthesize the best available evidence to guide clinician and patient decision making. There are a multitude of barriers and facilitators to clinicians adhering to CPGs; however, little is known about active cancer treatment CPG adherence specifically. This systematic review sought to identify clinician attitudes, and perceived barriers and facilitators to active cancer treatment CPG adherence.

Methods: A systematic search was undertaken of five databases; Ovid Medline, Psychlnfo, Embase, Scopus, CINAHL, and PROQUEST. The retrieved abstracts were screened for eligibility against inclusion criteria, and a full text review was conducted of all eligible studies. Data were extracted, and a quality assessment was conducted of all included studies. The qualitative papers were thematically analyzed. Attitudes, barriers, and facilitating factors extracted from the quantitative papers were categorized within the qualitative thematic framework.

Results: The search resulted in the identification of 9676 titles. After duplicates were removed, abstracts screened, and full texts reviewed, 15 studies were included. Four themes were identified which related to negative clinician attitudes and barriers to active cancer treatment CPG adherence: (1) concern over CPG content and currency of CPGs; (2) concern about the evidence underpinning CPGs; (3) clinician uncertainty and negative perceptions of CPGs; and (4) organizational and patient factors. The review also identified four themes related to positive attitudes and facilitators to active cancer treatment CPG adherence: (5) CPG accessibility and ease of use; (6) endorsement and dissemination of CPGs and adequate access to treatment facilities and resources; (7) awareness of CPGs and belief in their relevance; and (8) belief that CPGs support decision making, improve patient care, reduce clinical variation, and reduce costs.

\footnotetext{
* Correspondence: mia.bierbaum@hdr.mq.edu.au

${ }^{1}$ Australian Institute of Health Innovation (AlHI), Macquarie University, Level 6,

75 Talavera Road, Sydney, NSW 2019, Australia

Full list of author information is available at the end of the article
}

(c) The Author(s). 2020 Open Access This article is licensed under a Creative Commons Attribution 4.0 International License, which permits use, sharing, adaptation, distribution and reproduction in any medium or format, as long as you give appropriate credit to the original author(s) and the source, provide a link to the Creative Commons licence, and indicate if changes were made. The images or other third party material in this article are included in the article's Creative Commons licence, unless indicated otherwise in a credit line to the material. If material is not included in the article's Creative Commons licence and your intended use is not permitted by statutory regulation or exceeds the permitted use, you will need to obtain permission directly from the copyright holder. To view a copy of this licence, visit http://creativecommons.org/licenses/by/4.0/. The Creative Commons Public Domain Dedication waiver (http://creativecommons.org/publicdomain/zero/1.0/) applies to the data made available in this article, unless otherwise stated in a credit line to the data. 
(Continued from previous page)

Conclusion: These results highlight that adherence to active cancer treatment CPG recommendations by oncology clinicians is influenced by multiple factors such as attitudes, practices, and access to resources. The review has also revealed many similarities and differences in the factors associated with general CPG, and active cancer treatment CPG, adherence. These findings will inform tailored implementation strategies to increase adherence to cancer treatment CPGs.

Trial registration: PROSPERO (2019) CRD42019125748.

Keywords: Oncology, Clinical Practice Guidelines, Guideline adherent treatment, Implementation Science, Evidencebased practice

\section{Contributions to the literature}

- Implementation of Clinical Practice Guidelines (CPGs) is context specific; however, there has been no systematic assessment of the specific barriers and facilitators that apply to active cancer treatment CPG adherence.

- This manuscript systematically reviews relevant literature to identify clinician attitudes toward, and the perceived barriers and facilitators for adherence to active cancer treatment CPGs, and compares them to previously identified factors associated with general CPG adherence.

- The findings will inform targeted implementation of interventions to increase adherence to cancer treatment CPGs that overcome the context-specific barriers and utilize the identified facilitators.

\section{Background and objectives}

CPGs synthesize the best available evidence to guide clinician and patient decision making [1]. Evidence from published clinical trials is often interwoven with clinical practice insights derived through the consensus opinion of clinical experts [2, 3]. CPGs are typically developed by government bodies or professional organizations that undertake multidisciplinary consultation, and systematic review and synthesis of the latest evidence [2]. Ideally, the evidence is explicitly linked to the CPG recommendations, and the recommendations are updated in line with the latest evidence [4].

There is a spectrum of perceptions around the utility of CPGs in medicine. CPGs are heralded as a mechanism to reduce clinical practice variation, with the aim of improving patient outcomes [2, 5]. They are viewed by some clinicians as a way of minimizing intuitive, anecdotal, and potentially biased treatment decision making [6]. Other clinicians, however, are concerned by the potential for CPGs to restrict their autonomy, and perceive CPGs as impeding their ability to tailor treatment to patients' individual needs and preferences [7].
It has been noted that the production and dissemination of CPGs does not necessarily translate to the implementation of evidence into practice [2]. The implementation of CPGs often lags behind dissemination [8]. It has also been argued that the "uptake of research findings into routine health care is a haphazard and unpredictable process" (p. 107) [9] and barriers that impede the implementation of evidence translation arise at patient, clinician, organization, and policy levels [10].

Barriers to CPG adherence can be grouped within three domains; Clinician Awareness, Attitudes, and Work Practices regarding CPGs. A lack of clinician awareness of CPG recommendations [11] is a fundamental barrier to adherence which can be addressed by active dissemination rather than relying on simple diffusion [12].

\section{Negative attitudes toward CPGs also constitute important barriers}

CPGs have been criticized for their focus on explicit knowledge, rather than tacit, practice-based knowledge [3]. They elicit concerns that naive prescriptive guidelines lead to "cookbook medicine" [7, 13] (p. 504) disregarding the social and organizational context of knowledge sharing, in which medicine is practiced [3]. Negative attitudes toward CPGs [14] position them as "impractical," "rigid" tools that "reduce clinician autonomy," that are "intended to cut healthcare costs," while potentially increasing litigation for clinicians (p. 504) [13]. Concern that some CPGs are outdated [15] due to delays inherent in the development process, and concerns about the perceived quality of evidence underpinning CPGs [11], or use of misleading evidence, can also influence adherence [16].

Concerns have also been expressed about trial design and reporting biases [17] (with publication bias selecting for trials reporting significant results) [18], the evidence underpinning CPGs being based on clinical trials of healthier and younger patients who are unrepresentative of patients being treated in the real world (reducing 
CPG applicability) [19], and the influence of pharmaceutical companies on the treatment recommendations outlined in guidelines [20]. In response, there has been a call for CPG development to be more rigorous about the quality of evidence used, and to provide more refined tools to better guide implementation of recommendations [16].

\section{Clinician practices and care processes can influence their use of CPGs}

Experienced clinicians infrequently look at CPGs, particularly for familiar procedures, and may only review CPGs before meetings to amend policies or audit practice standards [3]. To solve complex clinical problems and source up-to-date information, clinicians often use alternate trusted information sources, such as other doctors, professional networks, conferences, and medical journals and magazines, trusting the evidence, rigor, and expertise of these sources and creating internal "mindlines" of "largely tacit knowledge" (p. 1013) [3]. A lack of resources and time to implement CPGs [14], and a lack of clinician motivation or clinical inertia of practice [7], can influence adherence, as can the complexity and ease of use of CPGs [11], patient preference [11], and "limited integration of guideline recommendations into organizational structures and processes" (p. 213).

The successful dissemination of CPGs requires strategies that enhance CPG awareness and provide easy access to guidelines and resources [12]. Use of multifaceted implementation support strategies such as education sessions, regular prompts and reminders, engagement with local opinion leaders, and the establishment of implementation teams, which has also been successful in enhancing the implementation of CPGs [21], particularly when strategies are tailored to address identified barriers [22]. The rates of adherence to CPGs vary across cancer streams and contexts [23-25]; tailoring of CPGs, and targeting of strategies, may offer potential remedies. Factors that facilitate CPG implementation include "positive staff attitudes and beliefs, leadership support, ...teamwork and collaboration, professional association support, and inter-organizational collaboration and networks." (p. 213) [14]. A systematic review found that positive clinician attitudes to CPGs frame them as "helpful", "educational tools", "intended to improve the quality of care". (p. 504) [13].

Involvement of the target group (e.g., surgeons) in CPG development has been found to enhance CPG implementation [11], and clinician age and experience also affects CPG use, with younger clinicians being more inclined to use CPGs than older or more experienced clinicians $[11,26,27]$. In addition, patient age has been found to influence the receipt of CPG adherent care for some cancer treatments, which may be related to tolerance of treatment, presence of comorbidities, or decisions regarding curative treatment [23].

In addition to the literature on CPG adherence in general, studies that have examined clinicians' attitudes toward cancer CPGs have found that clinicians perceive some cancer CPGs as lacking both clarity and alternative treatment strategies that cater for a full range of patient preferences [28]. For CPGs in general, some clinicians report concerns about the quality of evidence underpinning the recommendations in general cancer CPGs [28], and the impact of these CPGs on their professional autonomy [29] and authority [30]. In addition, cancer CPGs have elicited concerns about oversimplification [30], and some clinicians simply disagree with specific cancer CPGs [28]. Other factors such as patients' lack of health insurance [28] have been identified as a barrier to cancer CPG adherence, as has poor access to information technology (IT) or proficient IT skills [28]. It has been suggested that improved IT availability and access to CPGs via smartphone applications could facilitate use of cancer CPGs [28]. These studies also found that clinicians considered some cancer CPGs to be "convenient sources of advice," and "good educational tools," (p. 285) [30] that are intended to improve the quality of patient care $[28,30]$.

Despite this state of knowledge, there is currently a gap regarding the synthesis of clinicians' views around adherence to active cancer treatment CPGs, and the associated barriers and facilitators to CPG adherence. There is evidence that levels of adherence across a variety of cancer treatment CPGs is relatively low $[23-25,31-38]$. It is important to examine the reasons behind this, with a view to identifying potential improvements in design and content of CPGs, or their dissemination. There is evidence that CPGadherent treatments for an array of cancers are associated with higher survival rates [33, 39-42]; however, while CPG adherence is often used as a measure of quality of care, a lack of adherence does not necessarily represent suboptimal care, if there is reasonable justification for variation [43].

Clinicians' attitudes toward, and perceived barriers and facilitators for, adherence with active cancer treatment specific CPGs and CPGs in general are likely to overlap. However, the extent of the overlap is currently unknown. This systematic review aims to address the question: What are the attitudes of clinicians toward CPGs for active cancer treatment, and what are the perceived barriers and facilitators for adherence to these CPGs?

In this review, barriers refers to adherence obstacles specific to CPGs for active cancer treatment, and facilitators refers to enabling factors for adherence to those CPGs. 


\section{Research design and methods}

This systematic review was guided by the Preferred Reporting Items for Systematic Review and MetaAnalysis (PRISMA) statement [44], and registered on PROSPERO (2019) CRD42019125748.

\section{Eligibility criteria}

Quantitative, qualitative, and mixed methods studies were included in the review if they reported empirical evidence, were published in English in peer-reviewed journals, and examined clinicians' attitudes (including perceptions and views) toward, or perceived barriers and facilitators of adherence to CPGs focusing on active cancer treatment (excluding therapy with palliative intent) (Table 1). No publication date restrictions were applied. Studies were excluded if they focused on non-active cancer treatments, such as CPGs for screening, psychosocial care, symptom management, or cancer treatment with palliative intent.

\section{Types of participants and outcomes}

Study participants included clinicians who used cancer treatment CPGs to treat patients; this included clinicians potentially treating multiple tumor streams, such as radiation oncologists, medical oncologists, hematologists or general surgeons, and those treating single tumor streams, such as respiratory physicians. All data regarding clinicians' attitudes or perceived barriers or facilitators to active cancer treatment CPGs were included, including qualitative, mixed methods, and quantitative data.

\section{Search strategy}

A list of search terms (Table 2) was developed by the research team (MB, BNGE, GA) and reviewed with a medical librarian. Searches were performed in six selected databases (Ovid Medline, PsycINFO, Embase, Scopus, CINAHL, and PROQUEST) in November

Table 1 Eligibility criteria

Inclusion criteria:

1. Studies must include empirical research

2. Studies must be published in English

3. Studies must be published in a peer reviewed journa

4. Studies must report treating clinician attitudes towards CPGs for active cancer treatment or perceptions of barriers or facilitators to adherence to those CPGs.

Exclusion criteria

Articles not including empirical research were excluded. Studies reporting on CPGs that focused on other aspects of cancer care (such as screening, psychosocial care, palliative care, or symptom management CPGs) were excluded.
2018, and repeated to update the search with current literature in November 2019. These databases were selected to enable a broad search of the literature including biomedical science, behavioral science, humanities, healthcare, and nursing literature. Four groups of search terms were combined using keywords or Mesh terms.

\section{Study selection}

The citations and abstracts of titles identified in the searches were downloaded into Endnote and duplicates removed. The title abstracts were reviewed by four reviewers (MB, BNGE, $\mathrm{KH}, \mathrm{KL})$ to determine whether they met eligibility criteria (Table 1$)$. Three reviewers $(M B$, BNGE, KH) independently conducted a blind review of a sample of $5 \%$ of title abstracts to determine inter-rater reliability. All titles were reviewed by $\mathrm{MB}$, while $\mathrm{KL}, \mathrm{KH}$, and BNGE reviewed a $1 \%, 4 \%$, and $5 \%$ random sample of abstracts, respectively. Any disagreements were discussed after assessment by a separate reviewer (BNGE/ $\mathrm{KH})$ until consensus was reached. The full texts of the included eligible abstracts were then reviewed by $M B$, $\mathrm{BNGE}$, and $\mathrm{KH}$ to determine if they met the inclusion criteria. The rationale for inclusion or exclusion was recorded on a data extraction template. Reference lists of all included articles were searched for additional eligible articles.

\section{Data extraction}

Data were extracted from all included articles using a template which included location of study, study design, sample size, data collection method (e.g., interviews, surveys), survey response rate if applicable, cancer stream and stage, discipline of participants, career experience of participants (e.g., senior clinician, registrar), description of the CPG reported, and data that related to the review question, including the key barriers to CPG adherence, key facilitators to CPG adherence, attitudes to CPGs, and other details noted as important in the study. Data were extracted from all included studies by $\mathrm{MB}$, and a $5 \%$ sample of data extraction was confirmed by $\mathrm{KH}$ and BNGE.

\section{Quality assessment}

Quality assessment of each included article was conducted by $\mathrm{MB}$ and verified by BNGE and $\mathrm{KH}$ using the Mixed Methods Appraisal Tool (MMAT) [45], a reliable quality assessment tool used to assess the quality of mixed studies [46], utilizing its qualitative research and quantitative descriptive research sections [45]. 
Table 2 Primary search strategy

\begin{tabular}{|c|c|}
\hline Search terms & Limits \\
\hline $\begin{array}{l}\text { neoplasm* OR cancer* OR carcinoma* OR malignan* OR tumo?** OR oncology OR metastas* } \\
\text { AND } \\
\text { "practice guideline" OR "clinical practice" OR "clinical protocol" OR "evidence based practice" OR "Evidence } \\
\text { based medicine" OR guideline* OR "Practice pattern*" OR "clinical varia*" } \\
\text { AND } \\
\text { attitude* OR "Health personnel attitude" OR "Physician attitude" OR knowledge OR perspective* OR belief* OR } \\
\text { barrier* OR facilitat* OR implement* OR adheren* OR concordan* OR complian* } \\
\text { AND } \\
\text { physician OR clinician* OR surgeon OR "medical oncologist" OR radiologist OR doctor* OR registrar* OR } \\
\text { trainee* OR oncologist* }\end{array}$ & $\begin{array}{l}\text { Title/Abstract and/or subject } \\
\text { headings }^{\mathrm{a}, \mathrm{b}, \mathrm{c}, \mathrm{d}, \mathrm{e}, \mathrm{f}} \\
\text { English }^{\mathrm{a}, \mathrm{b}, \mathrm{d}, \mathrm{d}, \mathrm{d}, \mathrm{f}} \\
\text { Human } \\
\text { Not }(\text { conference abstract or } \\
\text { conference review) } \\
\text { Exclude reviews }^{\mathrm{b}, \mathrm{c}} \\
\text { Dissertations } \\
\text { only }^{\mathrm{a}}\end{array}$ \\
\hline
\end{tabular}

* Indicates truncation

aPROQUEST

${ }^{\mathrm{b}}$ Embase

'Scopus

${ }^{\mathrm{d}}$ Medline

ePsycINFO

fCINAHL

\section{Data analysis and synthesis}

The included studies used a range of methodologies, including qualitative and quantitative studies, rendering results heterogenous. Due to the heterogeneity of the questions asked in the survey-based quantitative studies, statistical aggregation was not appropriate. The included articles were read multiple times until $\mathrm{MB}$ was familiar with the content and context of the studies, before data extraction and coding began.

\section{Qualitative studies}

The results section of each qualitative paper was inductively coded, line by line, by MB using NVIVO version 12 [47]. The coding involved the designation and application of summarizing labels in order to identify the meaning of text components [48]. New codes were added to the code bank as new ideas were identified [49]. After all studies were coded, an overall, refined coding framework was finalized. The initial data-driven codes were then categorized into themes that represented recurring ideas throughout the studies relating to attitudes, and perceived barriers and facilitators to CPG adherence. Themes were checked by re-reviewing the articles, to ensure they accurately represented the data [49]. Coding from a random sample of six papers was confirmed by BNGE and $\mathrm{KH}$ (experienced qualitative coders), to ensure that the internal validity of coding was maintained. They verified the individual codes, coding patterns, and resulting thematic framework to ensure themes were grounded in the primary data, and checked for consistency and accuracy [49, 50]. The final themes were discussed and refined by the reviewing team, resulting in a final consensus-based thematic framework.

\section{Quantitative papers}

Data from the results section of each quantitative study were extracted using the data extraction sheet, documenting attitudes toward CPGs or perceived barriers and facilitators to CPG adherence. The heterogeneity of these studies led to a decision to describe the data extracted, rather than attempting aggregation of results from multiple studies. The themes identified in the quantitative studies were compared with the themes identified in the qualitative papers and these were found to align. Key attitudes, barriers, and facilitators from all papers were grouped under each overarching theme.

\section{Results}

Search process

The original and updated searches of the databases resulted in 10,159 title abstracts for review. Duplicate title abstracts were removed $(n=4153)$, leaving 6006 title abstracts to be screened. The level of agreement between the three reviewers ( $\mathrm{MB}, \mathrm{BNGE}$, and $\mathrm{KH}$ ) was calculated during a blinded $5 \%$ review of title abstracts: $98.4 \%$ agreement was achieved, with a Fleiss Kappa score of 0.64 [51]. KL also screened 5\% of titles abstracts screened by MB, which had $99 \%$ agreement and a Fleiss Kappa score of 0.80. Of the 6006 screened title abstracts, 5777 were excluded as they did not meet the eligibility criteria, while 229 were included for full text review. Complete agreement was achieved during full text review between $\mathrm{MB}$ and $\mathrm{KH}$, as well as between $\mathrm{MB}$ and $\mathrm{BNGE}$, resulting in 15 studies being included in the final analysis (see Fig. 1). All included studies were assessed for quality. No papers were excluded as a result of the quality assessment, to provide a comprehensive presentation 


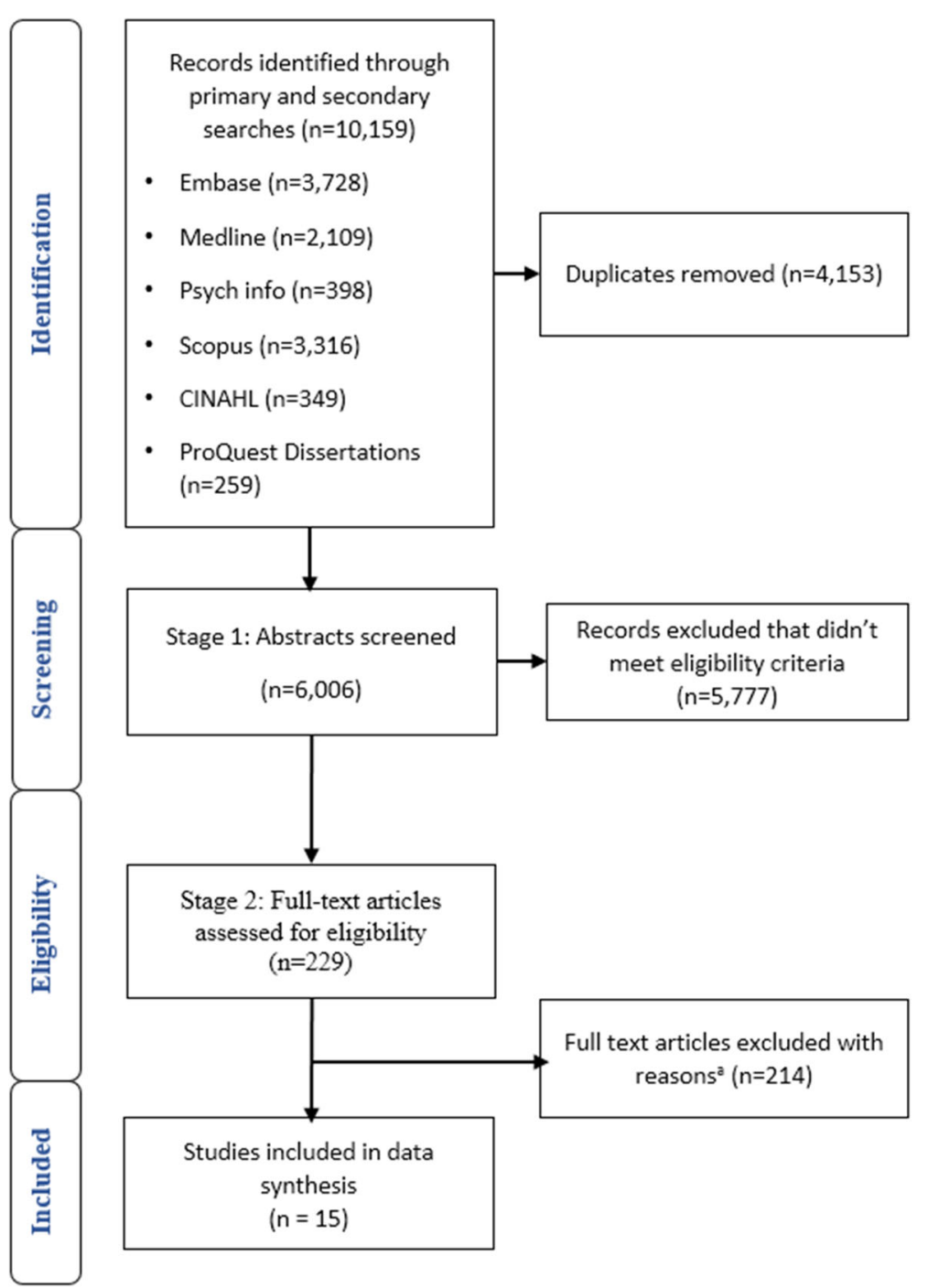

Fig. 1 PRISMA flow diagram of search strategy [52]

of the literature. The quality of the qualitative studies was high, while the quantitative studies were found to lack detail about nonresponse bias, and how representative the samples were of the target population (Table 3).

\section{Study characteristics}

The 15 included studies comprised three interviewbased qualitative studies [53-55], two studies that utilized qualitative and quantitative methods, and presented results of each method separately $[53,56]$, and 11 quantitative studies all using surveys [15, 5766]. Most studies were from Australia $(n=5)$ [58, $59,61,65,66]$ or Canada $(n=4)[15,54,56,57]$. Breast cancer CPGs were the most common focus $(n$
= 6) $[54,59,62,64-66]$. Study participants included radiation oncologists $(n=9)$, medical oncologists $(n$ $=9)$, and surgeons $(n=8)$, with most studies including multiple professional groups (Table 4). Three studies were published in the 1990s [59, 62, 65], two in the 2000s $[15,61]$, and ten from 2010 to 2019 $[53-58,60,63,64,66]$. It is also worth noting that only one study focused on CPG implementation in low- and middle-income countries, concluding that while awareness of cancer CPGs was high among clinicians, CPG implementation was limited by inadequate facilities and CPGs that were overly complex and not applicable to the local context [63]. The remaining studies were situated in high income countries (Table 4). 


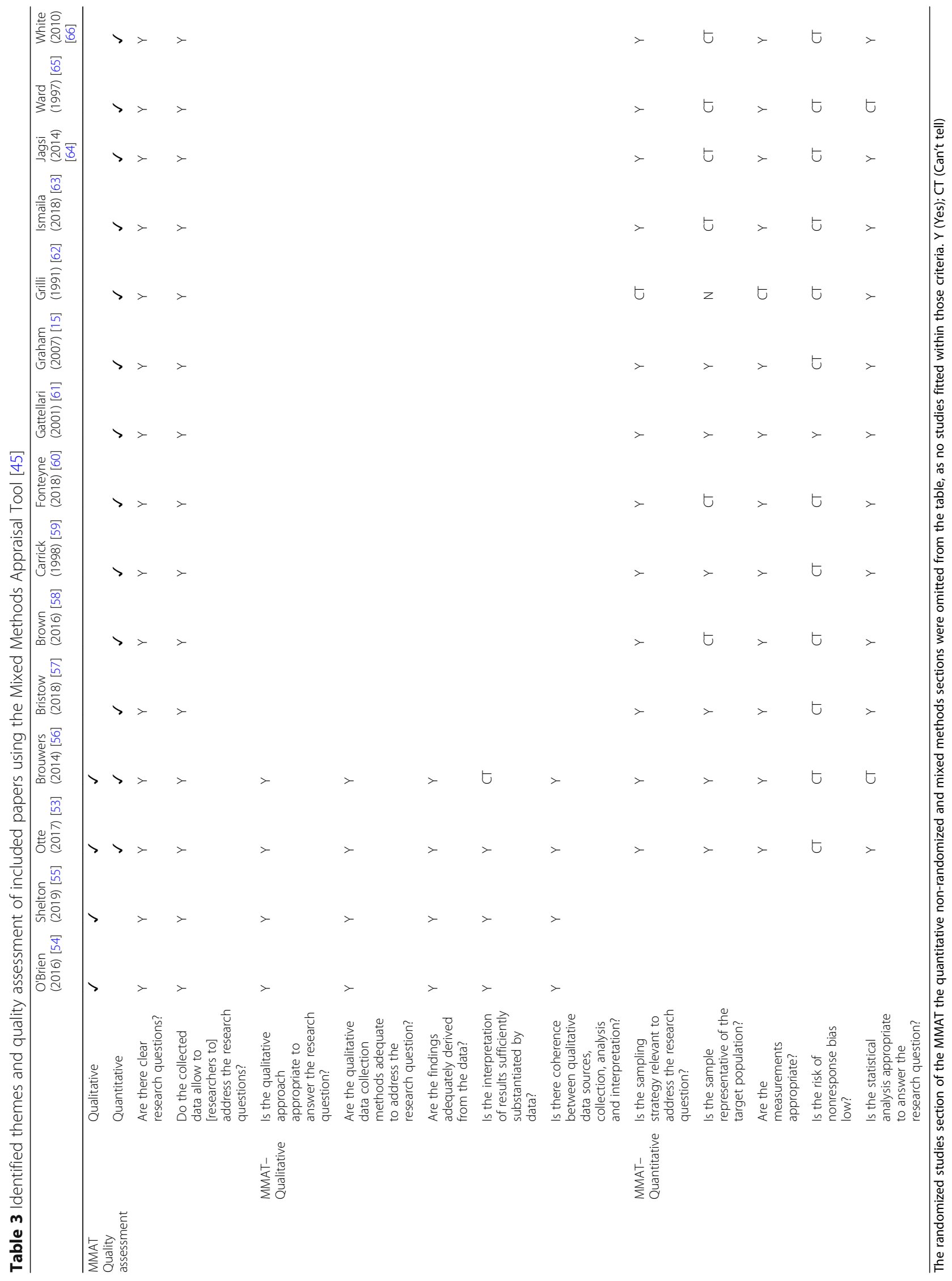




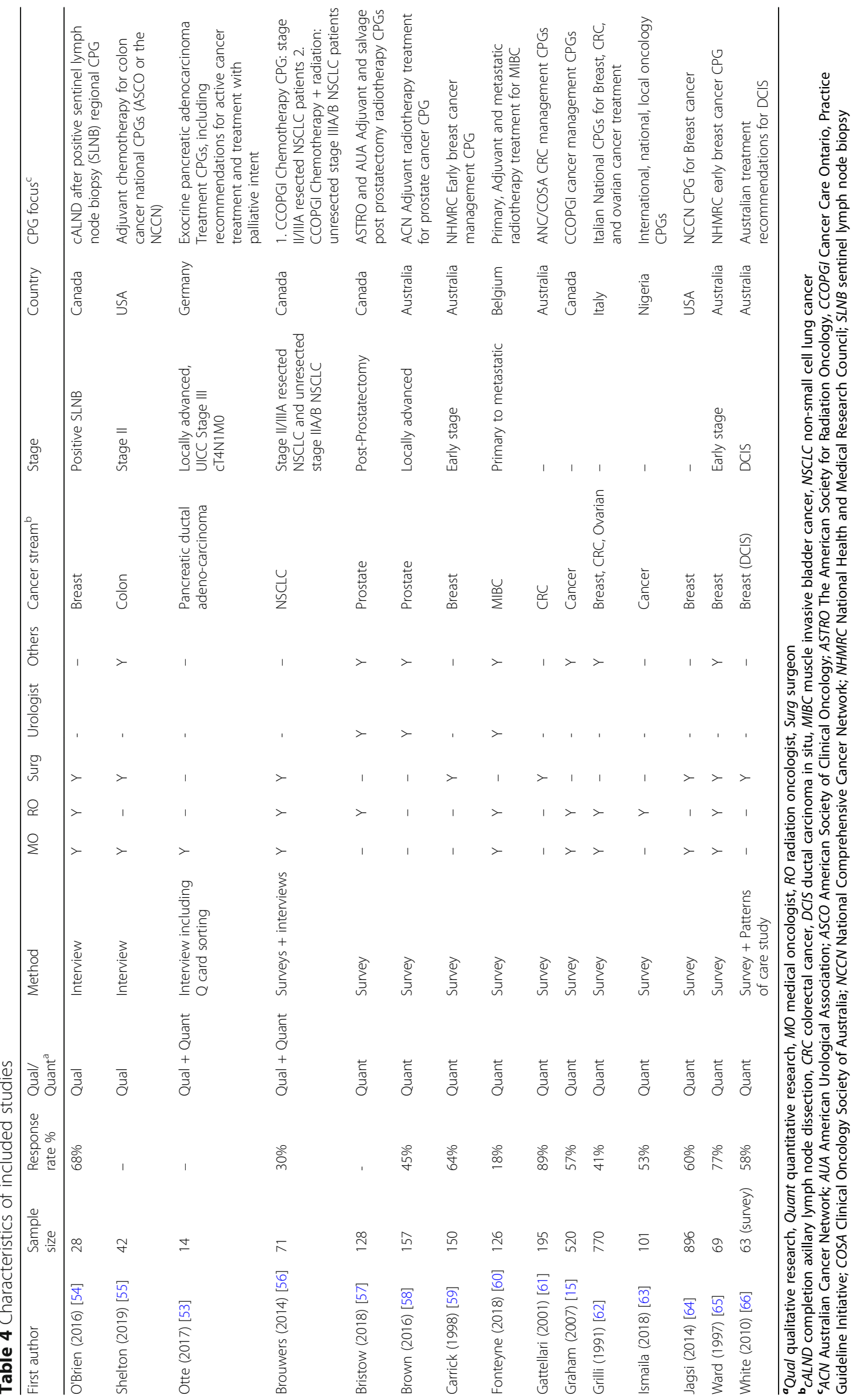




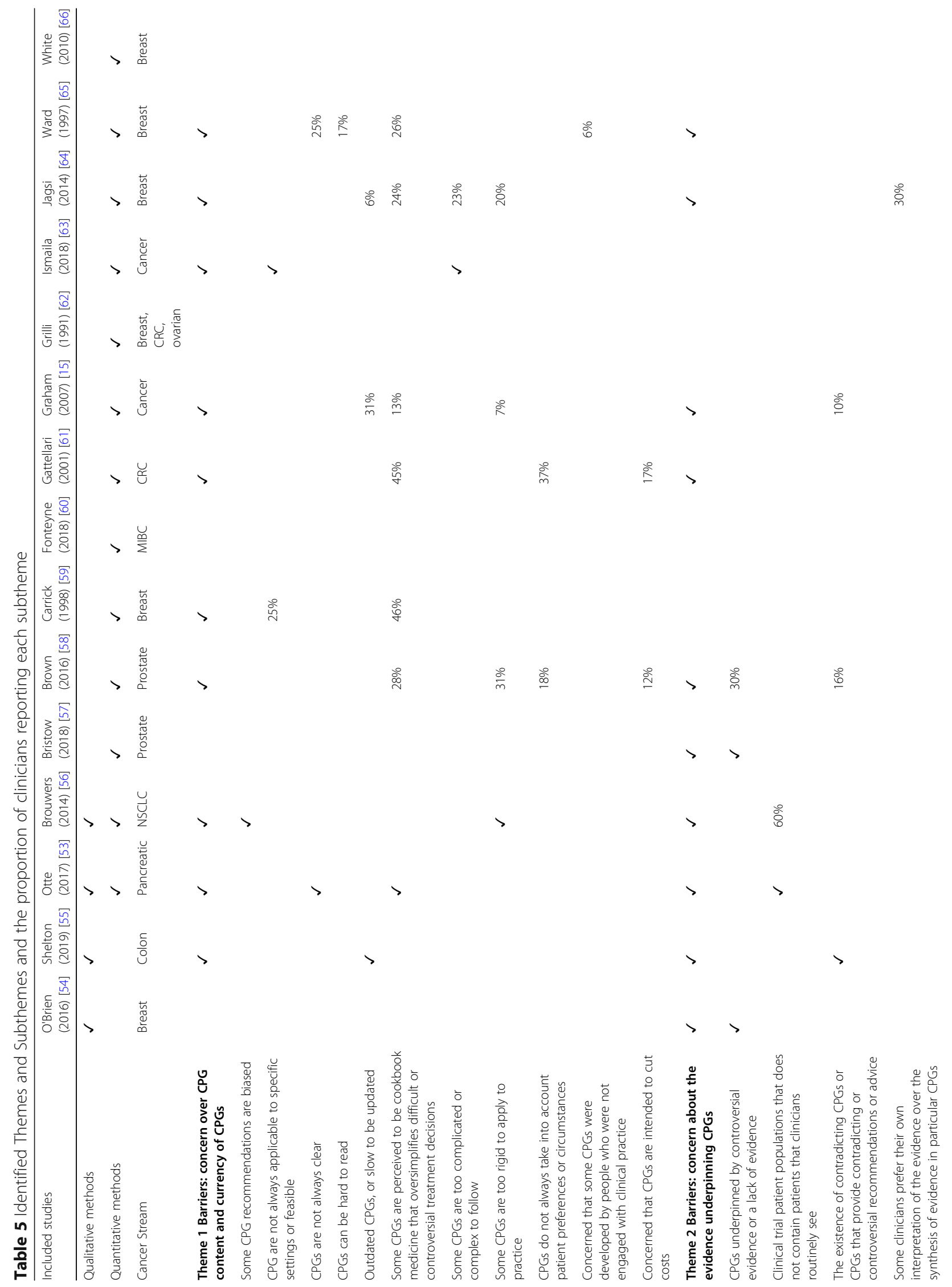




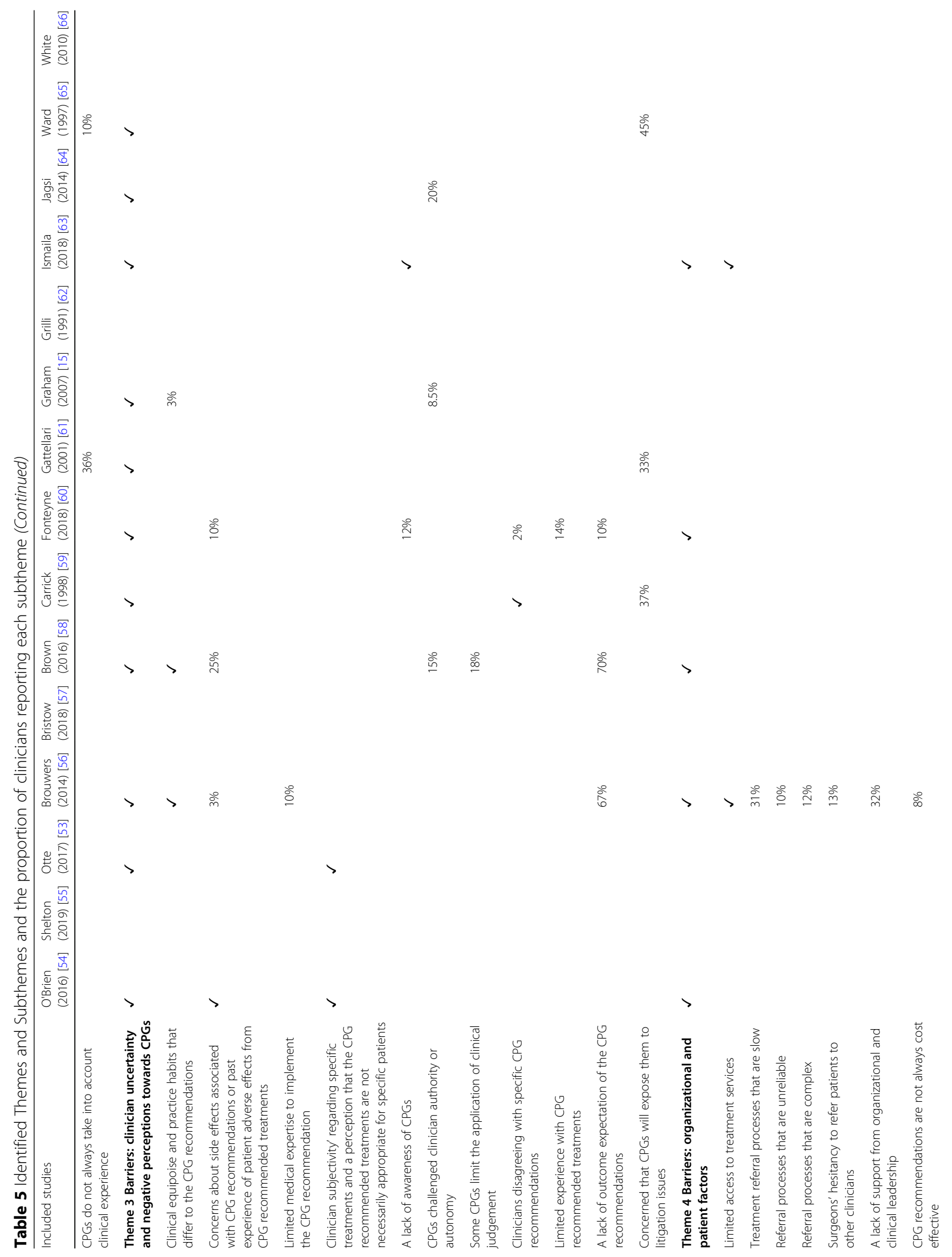




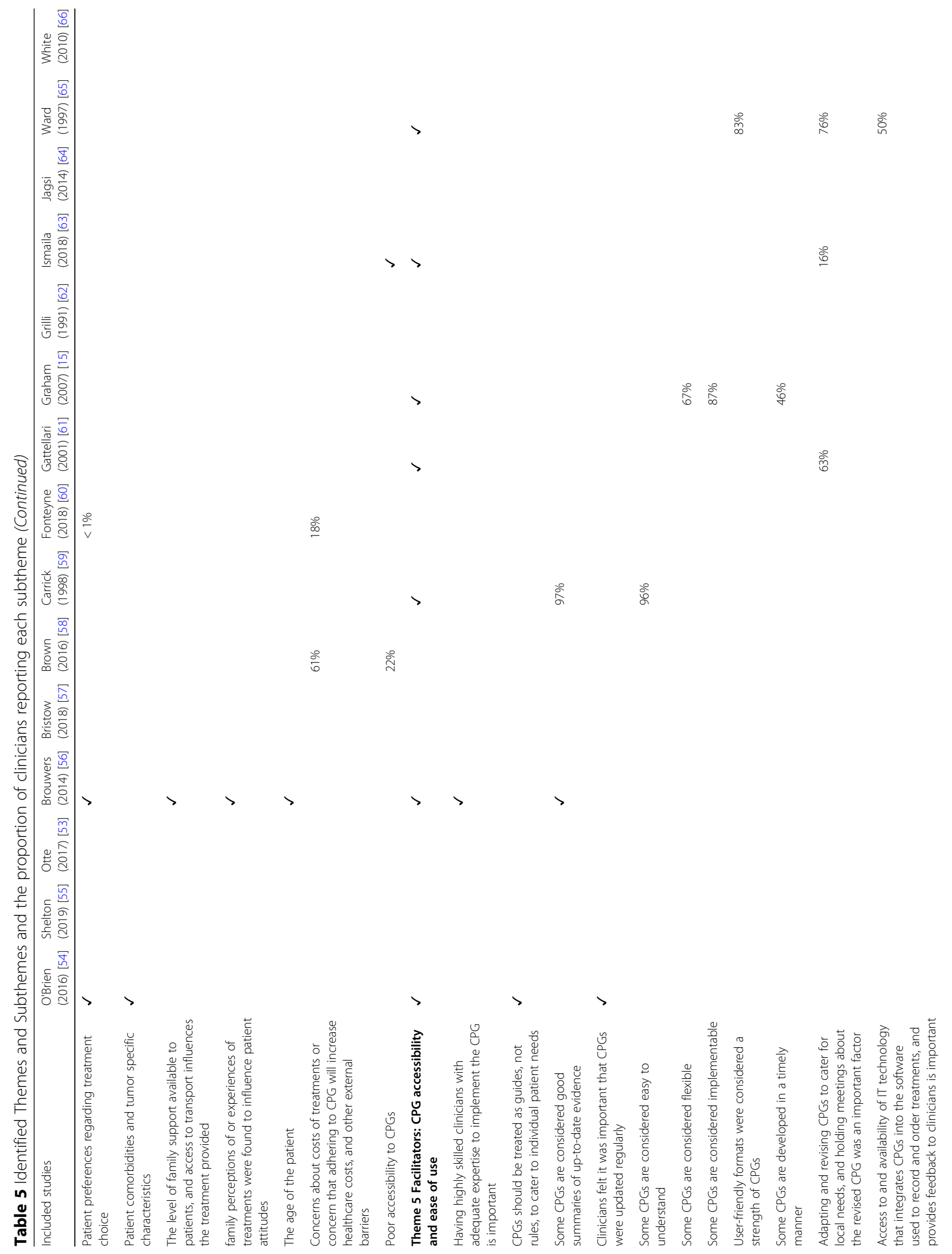




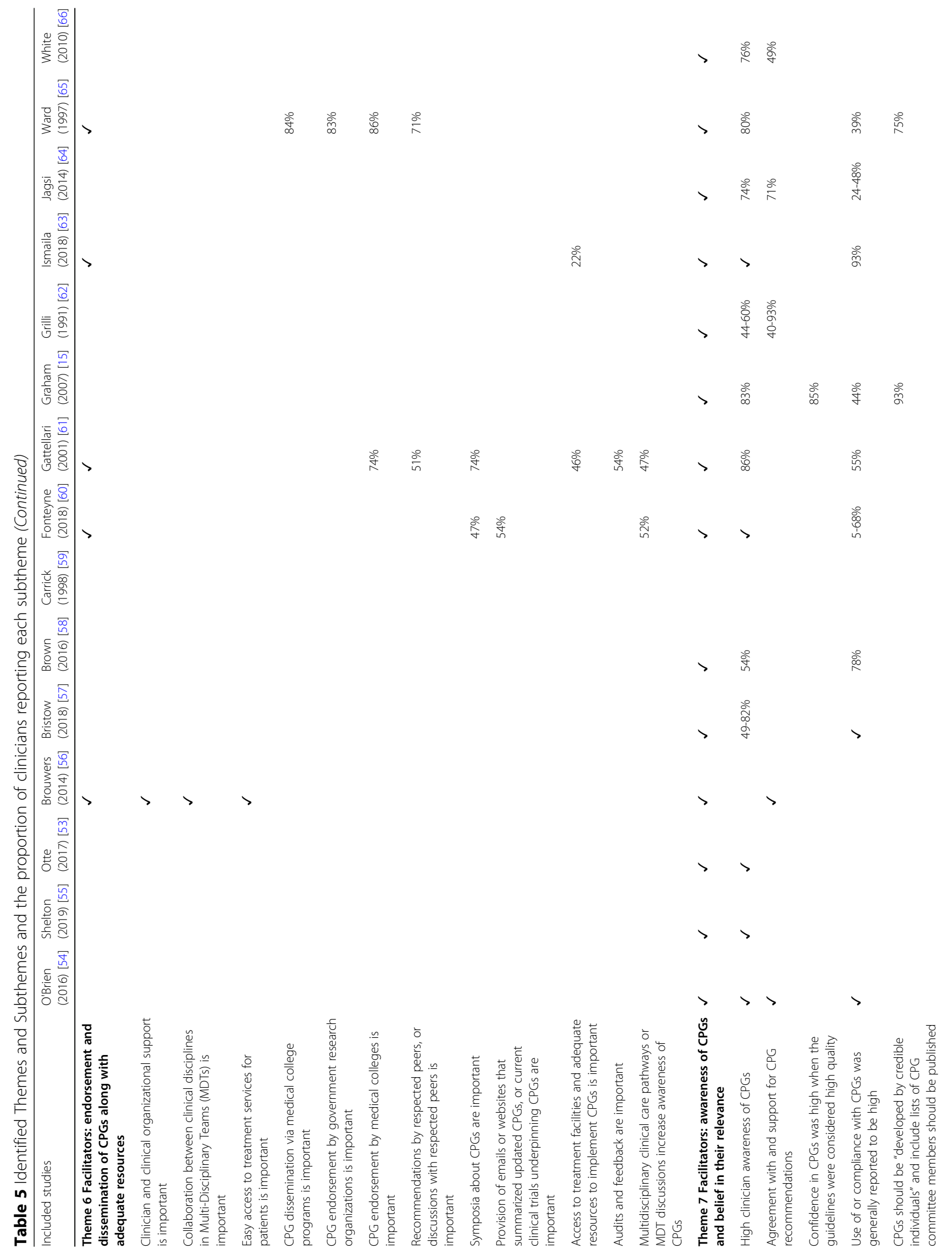




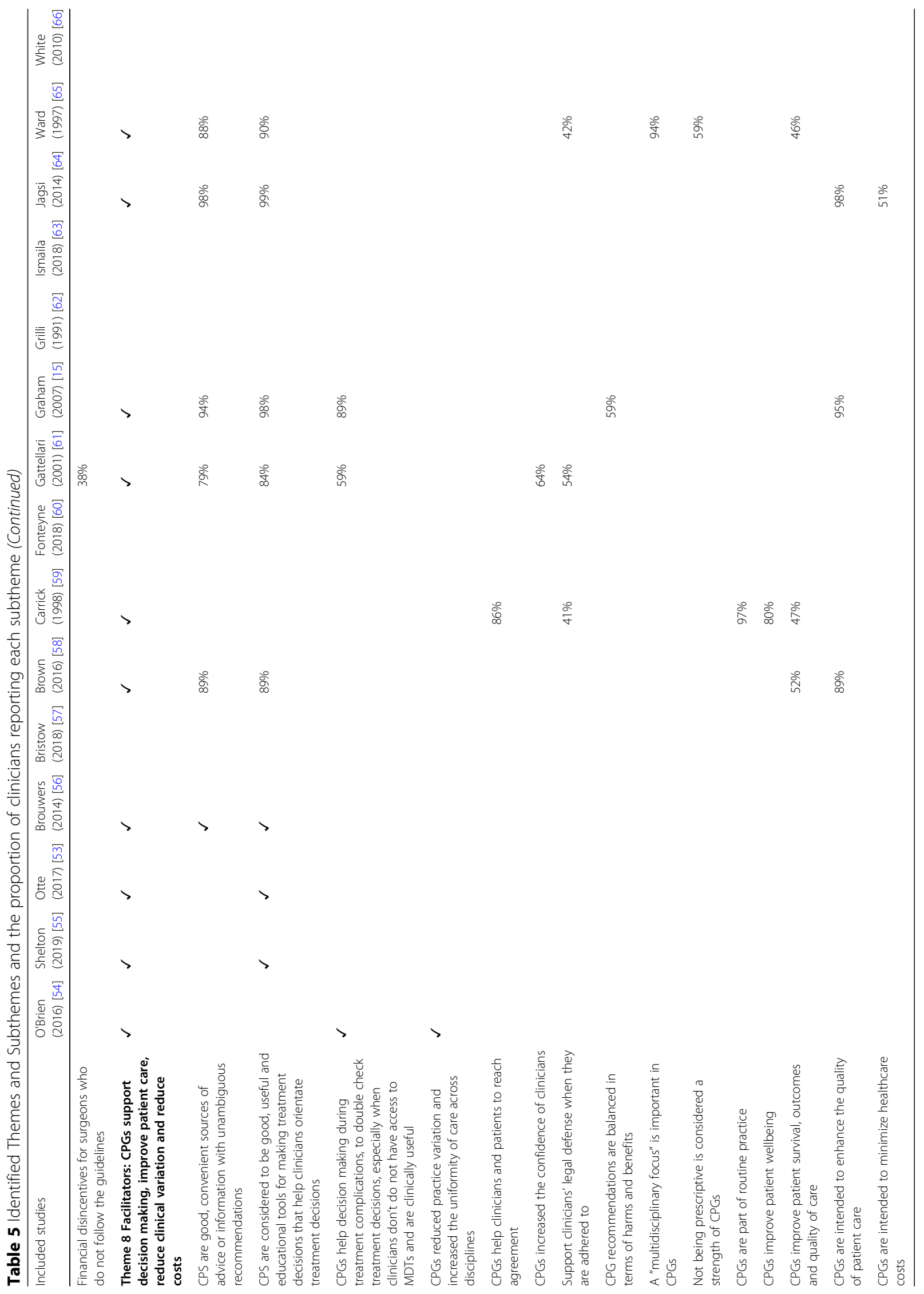




\section{Themes}

Four themes regarding negative attitudes and barriers to active cancer treatment CPG adherence were identified (themes 1-4), and four separate themes classified positive attitudes and facilitating factors to active cancer treatment CPG adherence (themes 58). Table 5 presents the themes, the proportion of clinicians reporting each factor, and the tumor stream focus.

\section{Negative attitudes and barriers}

\section{Theme 1: concern over CPG content and currency of cancer treatment CPGs}

Clinicians reported that some CPGs are not always applicable to specific settings [63], are not clear [65], are hard to apply [53], and hard to read [65]; all potential barriers to CPG adherence. It should be noted that one study [53] referred to CPGs for the treatment of locally advanced, UICC stage III cT4N1M0 pancreatic ductal adenocarcinoma, with recommendations for palliation as well as active cancer treatment. Clinicians thought some CPGs were slow to be updated [15], or were outdated $[55,64]$. Across five studies, a range of clinicians perceived that some CPGs can promote "cookbook medicine" $[15,58,59,61]$ (p. 150) [64], that is generic [53], and can oversimplify difficult or controversial treatment decisions [65].

Guidelines are very generic, which means they address certain age groups or patients that have benefited from a certain type of chemotherapy in a certain way. And this does not cover all the different factors ..., like patient preferences or social environment, sometimes the guidelines cover the age, but overall it is all very simplified.

Otte (2017) (p. 784) [53]

Other barriers to adherence included CPGs being too complicated or complex to follow [63, 64], or that the recommendations were not feasible [59]. In three studies, clinicians felt that some CPGs were too rigid to apply to practice $[56,58,64]$; however, the majority of clinicians in another study disagreed with that sentiment [15]. A small proportion of clinicians surveyed in one study felt that recommendations in a cancer CPG may be biased, which could also limit adherence [56].

Few clinicians agreed that CPGs take into account patient preferences or needs [58] or individual circumstances of patients [61]. A small number of clinicians were concerned that some CPGs were developed by people disengaged with clinical practice [65], and clinicians in two studies felt that CPGs were intended to cut costs $[58,61]$.
Theme 2: concern about the evidence underpinning cancer treatment CPGs

Clinicians raised concerns about the uncertainty generated by CPGs that contradict each other $[55,58]$ and felt that this contributed to the complexity of interdisciplinary decision making about treatment [54]. Clinicians also believed that some CPGs were underpinned by controversial $[15,54]$ or conflicting evidence [57], or a lack of evidence [58], which could also act as a barrier to adherence. Some clinicians in another study preferred their own interpretation of the evidence over the synthesis of evidence in particular CPGs [64]. Concerns were also raised that clinical trial patient populations from the studies underpinning some CPGs were not representative of the patients that clinicians routinely see $[53,56]$. Some clinicians felt that CPGs did not take into account clinical experience [61] and "emphasized published evidence to [the] detriment of clinical judgment" (p. 363) [65].

The patients who present in real life are much more variable with respect to functional status and comorbidities than the stage IIIA/ IIIB patients reflected in the evidence and PG recommendations. This lack of connection between the real life patient and the study patient can undermine the value, relevance and utility of the [Practice Guideline]. Brouwers (2014) (p. 43) [56]

Theme 3: clinician uncertainty and negative perceptions of cancer treatment CPGs

A few clinicians felt that CPGs challenged their authority [15] and autonomy [58, 64] by limiting their application of clinical judgment [58]. Clinical equipoise and habits that differed from the CPG recommendations were suggested barriers to CPG adherence [56, 58] and a small number of clinicians in one study felt that implementing a specific CPG would require too many changes to their practice [15]. Some clinicians reported disagreeing with specific CPG recommendations [59], and a minority felt that disagreeing with a CPG could be a barrier to adherence [60], noting that this study [60] did not differentiate between attitudes toward CPGs for radiotherapy in the primary, adjuvant, or metastatic (and potentially palliative) settings. A lack of awareness of CPGs was reported as a barrier by a small number of clinicians in two studies $[60,63]$.

A small number of clinicians raised clinician subjectivity regarding specific treatments for particular patients as a potential barrier to CPG adherence; some CPGrecommended treatments were perceived to be inappropriate for specific patients $[53,54]$. 
The concrete treatment recommendation physicians make to an oncologic patient depends highly on their subjective estimation of the patient's biological age and prognosis. Clinical guidelines are seen as an important point of reference, but cease being helpful in highly individual cases. Otte (2017) (p. 784) [53]

Some clinicians in three studies felt that the risk of side effects as a result of adhering to the CPGrecommended treatment was a barrier $[56,58,60]$, as well as limited medical expertise or clinician skill [56], or limited experience with the recommended treatment [60]. A lack of expectation of improved patient outcomes as a result of adhering to CPGs was another potential barrier reported in three studies $[56,58,60]$. A significant proportion of clinicians in three studies were concerned that CPGs could expose them to litigation [59, 61, 65], although some clinicians felt that CPGs would also protect them [59].

\section{Theme 4: organizational and patient factors}

A multitude of organizational barriers to CPG adherence were identified: limited access to treatment facilities and services [56, 63]; treatment referral processes that are slow, unreliable, and complex [56]; and a lack of support from organizational and clinical leadership [56]. A small proportion of clinicians also felt that surgeons' hesitancy to refer patients to other clinicians (like medical or radiation oncologists) was a barrier [56]. The costs of treatments was raised as a barrier in one study [60], while clinicians in other studies expressed concern that adhering to CPGs would increase healthcare costs [58] or that CPG recommendations were not always cost effective [56]. Poor access to CPGs in general was also identified as a factor that could limit CPG adherence [58, 63].

Patient preferences regarding treatment choice were perceived to limit adherence to CPG recommendations where these differ from CPG recommendations $[54,56,60]$. Patient comorbidities and tumor-specific characteristics were also found to limit clinicians' adherence to CPG recommendations if they perceived the treatments to be inappropriate [54]. The level of family support available to patients and patient access to transport were found to influence the treatments that clinicians offer [56], and family perceptions and experiences of treatments influenced patient attitudes [56]. The age of the patient was also mentioned as an influence on clinicians' choice of treatment in one study [56].

\section{Positive attitudes and facilitators}

Theme 5: cancer treatment CPG accessibility and ease of use

Theme 5 included factors that were seen to facilitate adherence to CPG recommendations. Clinicians were generally positive about cancer treatment CPGs, finding them easy to understand [59], flexible, and implementable [15]. CPG user-friendly formats were considered a strength of CPGs [65]. Having highly skilled clinicians with adequate expertise to implement a CPG was seen as important [56]. Clinicians felt that CPGs should be considered as guides, not rules, to allow flexibility to cater to individual patient needs [54], and they should contain up-to-date evidence [56] and be updated regularly [54]. Specific CPGs were considered applicable by a large proportion of clinicians in one study [56].

Guidelines, by definition, are simply guides, they are not protocols.' (S2) 'The guideline is not a cookiecutter for every patient.' (S11). O'Brien (2016) (p. 129) [54]

Many clinicians thought that specific CPGs were a good summary of the latest evidence $[15,59,65]$ and had been developed in a timely manner [15] while other CPGs were seen as providing an "unbiased synthesis" of the underpinning evidence [58, 61] (p. 151). It was considered important that CPGs cited the strength of evidence underpinning the recommendation $[61,65]$. Clinicians in one study were positive about the evidence underlying a specific CPG, finding the evidence base "complete," "convincing," "informative," "relevant," "strong," and "current." (p. 40) [56]. The majority of clinicians in another study valued CPGs that were based on randomized control trials and that provided detailed recommendations, preferring 9-10 years of follow-up evidence to convince them of the benefit of specific treatment options [58].

Adapting and revising CPGs to cater for local needs was an important factor that was seen to influence implementation and adherence [61, 63, 65] and holding meetings to locally adapt a CPG was considered an effective implementation strategy [61]. Access to, and availability of, IT technology that integrated CPGs into the software used to record and order treatments and provide feedback to clinicians was also reported to be an important implementation strategy [65].

\section{Theme 6: endorsement and dissemination of cancer treatment CPGs along with adequate access to treatment facilities and resources}

Most clinicians in one study reported that CPG dissemination via medical college programs, or other 
education related programs [65], as well as endorsement by government research organizations [65] or medical colleges $[61,65]$ were important strategies facilitating CPG adherence. Recommendations by respected peers [65], discussions about CPGs [61], and CPG symposia $[60,61]$ were also considered important facilitators.

Many clinicians suggested that the provision of emails or websites that summarized updated CPGs or current clinical trials underpinning CPGs were potential facilitators to enhance awareness of CPGs [60]. Access to treatment facilities with adequate resources to implement a CPG was identified as an effective facilitator to CPG use $[56,61,63]$, as was audit and feedback [61]. The presence of clinician and clinical organizational support were identified as facilitating factors of CPG adherence [56]. Multidisciplinary clinical care pathways [61], multidisciplinary team meeting (MDTM) discussions [60], and collaboration between clinical disciplines in multidisciplinary teams (MDTs) were suggested as ways to increase awareness of CPGs and support the decision making process [56].

\section{Theme 7: awareness of cancer treatment CPGs and belief in their relevance}

The vast majority of clinicians reported being aware of the CPGs each study focused on [54-56] with some variation in awareness $[15,57,58,60-66]$. In one study, awareness of CPGs was found to vary across disciplines, with radiation oncologists more aware of specific radiation therapy guidelines than urologists [57], and increasing clinician awareness of CPGs was identified as a facilitator to increase CPG usage (in low income countries) [63]. Agreement with CPG recommendations varied but was generally high $[62,64,66]$ and support for CPG recommendations was considered an important factor for adherence [56]. Confidence in CPGs was high when the guidelines were considered high quality [15]. Use of or compliance with CPGs was generally reported to be high $[15,57,58,60,63-65]$.

In one study, clinicians reported a variety of attributes of CPGs to be important, including the quality and level of evidence underpinning the CPGs, the "specification of the patient population to which a guideline is most applicable," the "strength of the recommendation," and the provision of cost effectiveness data (p. 151) [61]. Clinicians felt that CPGs should be "developed by credible individuals," (p. 611) [15] and that lists of CPG committee members should be published [65]. Some clinicians in one study felt that financial disincentives for surgeons who do not follow the guidelines would be effective strategies to facilitate adherence [61].
Theme 8: cancer treatment CPGs support decision making, improve patient care, reduce clinical variation, and reduce costs

CPGs were considered to be good, useful, and educational tools for making treatment decisions by most clinicians $[15,55,56,58,61,64,65]$.

Despite differences in reported use, most providers agreed that due to uncertainty regarding the benefits of [Adjuvant Chemotherapy] for this patient population, guidelines are important to help patients understand treatment options and to help providers make the most appropriate recommendation. Shelton (2019) (p. 287) [55]

CPGs were also considered to be "convenient sources of advice" or information $[15,56,58,61]$ (p. 151) $[64,65]$ that help clinicians orientate treatment decisions [53, 55] and help decision making during treatment complications [61]. CPGs were considered to be "safety nets" to double check treatment decisions, especially when clinicians do not have access to MDTs for peer consultation about treatment plans [54] (p. 128). Many clinicians in one study found that CPGs help clinicians and patients reach agreement [59], and clinicians in another study felt they increased the confidence of clinicians when making treatment decisions [61]. CPGs were also thought to support clinicians' legal defense, when adhered to [59, 61,65 and that the successful defense of a clinician who had practiced CPG adherent care would act as a facilitator for uptake of CPG recommendations by others [61].

Positive clinician attitudes toward CPG recommendations were found to be a strong predictor of CPG adherence [58]. Just under two-thirds of clinicians in one study felt that specific CPG recommendations were balanced in terms of harms and benefits, that the specific CPGs in question were very good to excellent quality, and that CPGs were useful [15]. The clinicians in that study were confident about the CPGs under discussion [15]. The "multidisciplinary focus" of a particular CPG was considered an important factor when deciding to adhere to the CPG [65] (p. 365 ) and not being prescriptive was also considered a strength of that CPG [65]. Clinicians in one study reported that CPGs were part of their routine practice [59]. CPGs are perceived by clinicians to improve patient wellbeing and survival [59], and patient outcomes and quality of care $[58,65]$ or are intended to enhance the quality of patient care $[15,58,64]$. Clinicians also felt that CPGs reduced practice variation and increased the uniformity of care across disciplines, enabling consistent treatment communication with patients [54]. Half of the clinicians in one 
Table 6 Comparison of previously identified factors and factors unique to cancer treatment CPG adherence

\author{
Previously identified factors $[7,11,13]$ mirrored in this review \\ Theme 1 Barriers: concern over CPG content and currency of CPGs \\ CPGs are "Biased" [7] (p. 1459) \\ CPGs lack "applicability to the practice population" [7] (p. 1460) \\ CPGs are "not easy to use" [7] (p. 1461) \\ CPGs are "Oversimplified and cookbook medicine" [7] (p. 1461), 13 \\ CPGs are "Cumbersome and confusing" [7] (p. 1461) \\ CPGs are "Impractical and too rigid to apply" [7] (p. 1459), 13
}

CPGs lack "credibility by guideline authors" [7] (p. 1460)

\section{Theme 2 Barriers: concern about the evidence underpinning CPGs}

Clinicians "disagreed with a guideline due to differences in interpretation of the evidence" [7] (p. 1460)

\section{Theme 3 Barriers: clinician uncertainty and negative perceptions towards CPGs}

Clinicians reported concern about: A "lack of motivation" to change routines and "Inertia of Previous Practice" [7] (p. 1459)

A lack of "outcome expectancy" [7] (p. 1461)

That CPG "benefits were not worth patient risk, discomfort or cost" [7] (p. 1460)

"A lack of self-efficacy" [7] (p. 1459)

A "lack of familiarity" and "awareness" of CPG [7] (p. 1459) 13

"Reduced autonomy" [7] (p. 1460) [13], would decrease flexibility [7]

A "lack of agreement" with the CPG [7] (p. 1460)

CPGs "will increased litigation or disciplinary action" [13] (p. 504)

\section{Theme 4 Barriers: organizational and patient factors}

Factors identified in this review

Some CPG recommendations are biased

CPG are not always applicable to specific settings or feasible

CPGs are not always clear

CPGs can be hard to read

Outdated CPGs, or slow to be updated

Some CPGs are perceived to lead to cookbook medicine that oversimplifies treatment decisions

Some CPGs are too complicated or complex to follow

Some CPGs are too rigid to apply to practice

CPGs do not always take into account patient preferences or circumstances

Some CPGs were developed by people not engaged with clinical practice

Concerned that CPGs are intended to cut costs

CPGs underpinned by controversial evidence or a lack of evidence

Clinical trial patient populations not reflective of the patients seen routinely by clinicians

Contradicting CPGs that provide contradicting or controversial recommendations or advice

Preference for own interpretation of the evidence over the synthesis of evidence in CPGs

CPGs do not always take into account clinical experience

Clinical equipoise and practice habits that differ to the CPG recommendations

A lack of outcome expectation of the CPG recommendations

Concerns about side effects associated with CPG recommendations

Experience of patient adverse effects from CPG treatments

Limited medical expertise to implement the CPG recommendation

A perception that the CPG treatments are not necessarily appropriate for specific patients

A lack of awareness of CPGs

CPGs challenged clinician authority or autonomy

Some CPGs limit the application of clinical judgment

Clinicians disagreeing with specific CPG recommendations

Limited experience with CPG recommended treatments

Concerned that CPGs will expose them to litigation issues

Limited access to treatment services

Treatment referral processes that are slow

Referral processes that are unreliable

Referral processes that are complex

Surgeons' hesitancy to refer patients to other clinicians 
Table 6 Comparison of previously identified factors and factors unique to cancer treatment CPG adherence (Continued)

Previously identified factors $[7,11,13]$ mirrored in this review

Clinicians reported barriers to adherence including "Patient factors" or characteristics [7] (p. 1459) [11], which may include factors like Patient preferences regarding treatment, Patient comorbidities and tumor specific characteristics [11]
Factors identified in this review

A lack of support from organizational and clinical leadership

CPG recommendations are not always cost effective

Patient preferences regarding treatment choice

Patient comorbidities and tumor specific characteristics

The level of family support available to patients, and access to transport influences the treatment provided

Family perceptions of or experiences of treatments were found to influence patient attitudes

The age of the patient

Concerns that costs of treatments or concern that adhering to CPG will increase healthcare costs, and other external barriers

Poor accessibility to CPGs

\section{Theme 5 Facilitators: CPG accessibility and ease of use}

CPGs that are evidence based are more likely to be adhered to [11]

Easy to use CPGs were more likely to be followed, if they don't require specialized resources and can be easily trialed [11]

Having highly skilled clinicians with adequate expertise to implement the CPG is important

CPGs should be thought of as guides

Some CPGs are considered good summaries of up-to-date evidence

Some CPGs are considered easy to understand
Some CPGs are considered flexible

Some CPGs are considered implementable

User-friendly formats were considered a strength of CPGS

Some CPGs are developed in a timely manner

CPGs should be updated regularly

Adapting and revising CPGs to cater for local needs, and holding meetings about the revised CPG is an important factor

Access to and availability of IT technology that integrates CPGs into the software used to record and order treatments, and provides feedback to clinicians is important

\section{Theme 6 Facilitators: endorsement and dissemination of CPGs along with adequate resources}

Clinician and clinical organizational support are important

Collaboration between clinical disciplines in Multi-Disciplinary Teams (MDTs) is important

Easy access to treatment services for patients is important

CPG dissemination via medical college programs is important

CPG endorsement by government research organizations is important

CPG endorsement by medical colleges is important

Recommendations by respected peers, or discussions with respected peers is important

Symposia about CPGs are important

Provision of emails or websites that summarized updated CPGs, or current clinical trials underpinning CPGs are important

Access to treatment facilities and adequate resources to implement CPGs is important 
Table 6 Comparison of previously identified factors and factors unique to cancer treatment CPG adherence (Continued)

Previously identified factors $[7,11,13]$ mirrored in this review
Factors identified in this review

Audits and feedback are important

Multidisciplinary clinical care pathways or MDT discussions increase awareness of CPGs

High clinician awareness of CPGs

Agreement with and support for CPG recommendations

Confidence in CPGs was high when the guidelines were considered high quality

Use of or compliance with CPGs was generally reported to be high

CPGs should be "developed by credible individuals" and include lists of CPG committee members should be published

Financial disincentives for surgeons who do not follow the guidelines

\section{Theme 8 Facilitators: CPGs support decision making, improve patient care, reduce clinical variation and reduce costs}

CPGs were considered to be "helpful sources of advice" and information [13] (p. 504)

CPGs were considered to be "good educational tools" for making treatment decisions [13] (p. 504)
CPS are good, convenient sources of advice or information with unambiguous recommendations

CPS are considered to be good, useful and educational tools for making treatment decisions that help clinicians orientate treatment decisions

CPGs help decision making during treatment complications, to double check treatment decisions, especially when clinicians don't do not have access to MDTs

CPGs reduced practice variation and increased the uniformity of care across disciplines

CPGs help clinicians and patients to reach agreement

CPGs increased the confidence of clinicians

Support clinicians' legal defense when they are adhered to

CPG recommendations are balanced in terms of harms and benefits

CPGs are clinically useful

A "multidisciplinary focus" is important in CPGs

Not being prescriptive is considered a strength of CPGs

CPGs are part of routine practice

CPGs improve patient wellbeing

CPGs improve patient survival, outcomes and quality of care

CPGs are intended to enhance the quality of patient care

CPGs are intended to minimize healthcare costs identified four themes centered around negative attitudes and barriers, and another four focused on positive attitudes and facilitators.

These results highlight diversity in clinician views about CPGs. This may be related to variety in the quality of the guidelines, and associated evidence, being discussed in each study. One recurring theme was the lack of clinician trust in the evidence underpinning the CPGs. High-quality guidelines include details regarding the qualitative and quantitative research. The review 
level of evidence underpinning each recommendation, identified through systematic review, or expert consensus, whereas poorer quality guidelines may not include that degree of detail [67-69]. This could explain clinician uncertainty regarding the evidence base, and the lack of outcome expectancy from adhering to CPGs, identified in the review. Infrequently updated CPGs may also contribute to these concerns [15], if they are underpinned by outdated evidence, as well as concerns about clinical trial publication bias [18].

Another clinician concern was that the evidence underpinning CPGs was based on clinical trials with cohorts of patients that were healthier or younger than the patients being treated, reflecting concern that this may invalidate the guidelines. While ideally CPGs would cater for all patient types, it is an inherent limitation that CPGs can only provide recommendations for patient cohorts, for which there is evidence to support treatments. This concern may be highlighting a need for greater clinical trial evidence regarding the efficacy of treatments in patients with poorer health status, older age, or comorbidities. The applicability of CPGs may be strengthened if realworld data sources (e.g., electronic health records) with more representative samples of patients [70] are incorporated into the evidence-base that underpins CPG recommendations, especially for patients who fit outside the study population of the original randomized trials.

In non-cancer specific literature, clinician experience or age were found to influence adherence to CPGs in general, with one review finding that less experienced clinicians were more likely to adhere to CPG recommendations than senior clinicians [11]. This factor was not identified in the present review, but has been found in a study looking at more general cancer related CPG adherence [71]. This may reflect the patient populations seen, with more experienced clinicians disproportionately treating more complex cases.

There were many attitudes, barriers, and facilitators identified in themes $1-4,5$, and 8 that overlapped with previously identified barriers and facilitators to general CPG adherence $[7,11,13]$ (Table 6). This review also identified additional attitudes, barriers, or facilitators to active cancer treatment CPG adherence, specifically. Themes 6 and 7 solely identified factors that were specific to cancer treatment CPG adherence (Table 6).

These results highlight that adherence to cancer treatment CPG recommendations by oncology clinicians is influenced by multiple interlinked factors such as attitudes, practices, resouces available, and support provided by organisatoins [72]. It is important that cancer treatment CPG implementation strategies are multifaceted, and target patients, clinicians, organizations, and policy [10], taking into account the social and organizational structures that influence implementation, and ensuring that they are tailored to the local context [3]. These factors that are unique to cancer CPG adherence, also reflect the multi-disciplinary nature of modern cancer treatment, and the fact that many clinicians are involved in treating multiple different types of cancers and are therefore exposed to multiple CPGs. Similarly, they may reflect the fast pace development of cancer research, and the associated challenges with maintaining up to date CPGs, as well as the complexity of tailoring treatments to individual patient needs.

\section{Strengths and limitations Limitations}

This review restricted the inclusion criteria to studies regarding CPGs for active cancer treatment, which meant that CPGs focusing on an array of other key issues (e.g., prevention and screening, symptom management, psycho-social care, and palliative care) were excluded. The study also restricted the inclusion criteria to treating clinicians' attitudes and perceived barriers and facilitators, which meant that studies that also included clinicians from other disciplines, such as psychologists and policy makers, were excluded if the attitudes of non-treating clinicians were not reported separately [73]. The review also restricted the criteria to only include studies published in English.

\section{Strengths}

This review consolidated knowledge about attitudes, barriers and facilitators that influence adherence to cancer treatment CPGs. While reviews conducted in past decades have identified barriers, facilitators or attitudes toward CPGs in general, this current systematic review is the first to combine all three facets, specifically targeting adherence to CPGs for active cancer treatment.

\section{Conclusion}

We examined and thematized clinician attitudes to, and perceived barriers to and facilitators of, adherence to CPGs for active cancer treatment. The review has drawn attention to the many similarities and some differences in the factors associated with general CPG, and cancer treatment CPG, adherence. These findings will inform tailored implementation strategies to increase adherence to cancer treatment CPGs by overcoming specific barriers, considering the local context and utilizing the cancer treatment-specific facilitators, while being cognizant of the oncologyspecific attitudes identified toward cancer treatment CPGs. 


\section{Appendix}

Table 7 PRISMA checklist

\begin{tabular}{lll}
\hline Section/topic & $\#$ & Checklist item \\
\hline $\begin{array}{l}\text { Title } \\
\text { Title }\end{array}$ & 1 & Identify the report as a systematic review, meta-analysis, or both. \\
$\begin{array}{l}\text { Abstract } \\
\text { Structured summary }\end{array}$ & $2 \begin{array}{l}\text { Provide a structured summary including, as applicable: background; objectives; data sources; study eligibility } \\
\text { criteria, participants, and interventions; study appraisal and synthesis methods; results; limitations; } \\
\text { conclusions and implications of key findings; systematic review registration number. }\end{array}$
\end{tabular}

Introduction

Rationale

3 Describe the rationale for the review in the context of what is already known.

Objectives

4 Provide an explicit statement of questions being addressed with reference to participants, interventions,

Methods comparisons, outcomes, and study design (PICOS).

Protocol and registration

Eligibility criteria

Information sources

Search

Study selection

Data collection process

Data items

Risk of bias in individual studies

Summary measures

Synthesis of results

Section/topic

Risk of bias across studies

Additional analyses

Results

Study selection

Study characteristics

Risk of bias within studies

Results of individual studies

Synthesis of results

Risk of bias across studies

Additional analysis
5 Indicate if a review protocol exists, if and where it can be accessed (e.g., Web address), and, if available, provide registration information including registration number.

6 Specify study characteristics (e.g., PICOS, length of follow-up) and report characteristics (e.g., years considered, language, publication status) used as criteria for eligibility, giving rationale.

7 Describe all information sources (e.g., databases with dates of coverage, contact with study authors to identify additional studies) in the search and date last searched.

8 Present full electronic search strategy for at least one database, including any limits used, such that it could be repeated.

9 State the process for selecting studies (i.e., screening, eligibility, included in systematic review, and, if applicable, included in the meta-analysis).

10 Describe method of data extraction from reports (e.g., piloted forms, independently, in duplicate) and any processes for obtaining and confirming data from investigators.

11 List and define all variables for which data were sought (e.g., PICOS, funding sources) and any assumptions and simplifications made.

12 Describe methods used for assessing risk of bias of individual studies (including specification of whether this was done at the study or outcome level), and how this information is to be used in any data synthesis.

13 State the principal summary measures (e.g., risk ratio, difference in means).

14 Describe the methods of handling data and combining results of studies, if done, including measures of consistency (e.g., $\left.{ }^{2}\right)$ for each meta-analysis.

\# Checklist item

15 Specify any assessment of risk of bias that may affect the cumulative evidence (e.g., publication bias, selective reporting within studies)

16 Describe methods of additional analyses (e.g., sensitivity or subgroup analyses, meta-regression), if done, indicating which were pre-specified.

17 Give numbers of studies screened, assessed for eligibility, and included in the review, with reasons for exclusions at each stage, ideally with a flow diagram.

18 For each study, present characteristics for which data were extracted (e.g., study size, PICOS, follow-up period) and provide the citations.

19 Present data on risk of bias of each study and, if available, any outcome level assessment (see item 12).

20 For all outcomes considered (benefits or harms), present, for each study: (a) simple summary data for each intervention group (b) effect estimates and confidence intervals, ideally with a forest plot.

21 Present results of each meta-analysis done, including confidence intervals and measures of consistency.

22 Present results of any assessment of risk of bias across studies (see Item 15).

23 Give results of additional analyses, if done (e.g., sensitivity or subgroup analyses, meta-regression [see Item 16]).
$6-10$

15,39

11

11-12 
Table 7 PRISMA checklist (Continued)

\begin{tabular}{|c|c|c|c|}
\hline Section/topic & $\#$ & Checklist item & $\begin{array}{l}\text { Reported } \\
\text { on page \# }\end{array}$ \\
\hline \multicolumn{4}{|l|}{ Discussion } \\
\hline Summary of evidence & 24 & $\begin{array}{l}\text { Summarize the main findings including the strength of evidence for each main outcome; consider their } \\
\text { relevance to key groups (e.g., healthcare providers, users, and policy makers). }\end{array}$ & $25-27$ \\
\hline Limitations & 25 & $\begin{array}{l}\text { Discuss limitations at study and outcome level (e.g., risk of bias), and at review-level (e.g., incomplete re- } \\
\text { trieval of identified research, reporting bias). }\end{array}$ & 27 \\
\hline Conclusions & 26 & $\begin{array}{l}\text { Provide a general interpretation of the results in the context of other evidence, and implications for future } \\
\text { research. }\end{array}$ & 28 \\
\hline \multicolumn{4}{|l|}{ Funding } \\
\hline Funding & 27 & $\begin{array}{l}\text { Describe sources of funding for the systematic review and other support (e.g., supply of data); role of } \\
\text { funders for the systematic review. }\end{array}$ & 30 \\
\hline
\end{tabular}

\section{Abbreviations}

ACN: Australian Cancer Network; ASCO: American Society of Clinical Oncology; ASTRO: The American Society for Radiation Oncology; AUA: American Urological Association; CALND: Completion axillary lymph node dissection; CCOPGl: Cancer Care Ontario, Practice Guideline Initiative; COSA: Clinical Oncology Society of Australia; CPGs: Clinical Practice Guidelines; CRC: Colorectal cancer; CT: Can't tell; DCIS: Ductal carcinoma in situ; IT: Information technology; MDTs: Multi-disciplinary teams; MDTM: Multidisciplinary team meeting; MIBC: Muscle invasive bladder cancer; MMAT: Mixed Methods Appraisal Tool; MO: Medical oncologist; NCCN: National Comprehensive Cancer Network; NHMRC: National Health and Medical Research Council; NSCLC: Non-small cell lung cancer; PRISMA: Preferred Reporting Items for Systematic Reviews and MetaAnalyses; PROSPERO: PROSPERO: International prospective register of systematic reviews; RO: Radiation oncologist; SLNB: Sentinel lymph node biopsy; Surg: Surgeon

\section{Acknowledgements}

None.

\section{Authors' contributions}

$M B$ conceptualized the study and produced the first draft of the manuscript. FR, GA, BNGE, and JB reviewed the study design and provided feedback, while $G D, W L, R K$, and $I O$ provided clinical advice regarding the study design, and the findings. $M B$ and $G A$ devised the search strategy, which was carried out by $\mathrm{MB} . \mathrm{MB}, \mathrm{BNGE}, \mathrm{KL}$, and $\mathrm{KH}$ completed the blinded $5 \%$ review of abstracts. BNGE and $\mathrm{KH}$ reviewed an additional $5 \%$ of the abstracts. MB reviewed all of the abstracts. MB carried out the full text review, with a $5 \%$ sample validated by both BNGE and $\mathrm{KH}$. This was followed by the data extraction and quality assessment of included articles by MB. BNGE and $\mathrm{KH}$ validated a sample of data extraction and quality assessment. MB developed the initial categorization frameworks with BNGE and $\mathrm{KH}$ providing feedback. $\mathrm{MB}$ completed the remainder of the data analysis including coding frameworks. BNGE and $\mathrm{KH}$ validated the analysis by confirming that it was grounded in the data. All authors contributed to revisions of subsequent drafts of the manuscript and approved the final submission.

\section{Funding}

This work was supported by an Australian Government's Research Training Program Scholarship associated with the Australian Institute of Health Innovation, Macquarie University, ID:9100002 awarded to MB.

\section{Availability of data and materials}

Data sharing is not applicable to this article as no datasets were generated or analyzed during the current study.

Ethics approval and consent to participate Not applicable.

\section{Consent for publication}

Not applicable.

\section{Competing interests}

The authors declare that they have no competing interests.

\begin{abstract}
Author details
${ }^{1}$ Australian Institute of Health Innovation (AlHI), Macquarie University, Level 6, 75 Talavera Road, Sydney, NSW 2019, Australia. ${ }^{2}$ Centre for Research Excellence in Implementation Science in Oncology, AlHI, Macquarie University, Sydney, Australia. ${ }^{3}$ Cancer Services, South Western Sydney Local Health District Cancer Services, Sydney, Australia. ${ }^{4}$ University of NSW, Sydney, Australia. ${ }^{5}$ Ingham Institute of Applied Medical Research, Liverpool, Australia. ${ }^{6}$ South Eastern Sydney Local Health District Cancer Services, Kogarah, Australia. ${ }^{7}$ Department of Clinical Medicine, Macquarie University, Sydney, Australia. ${ }^{8}$ University of Adelaide, Adelaide, Australia.
\end{abstract}

Received: 10 December 2019 Accepted: 14 April 2020 Published online: 27 May 2020

\section{References}

1. Grol R. Successes and failures in the implementation of evidence-based guidelines for clinical practice. Medical Care. 2001:||46-54.

2. Harrison M, Légaré F, Graham I, Fervers B. Adapting clinical practice guidelines to local context and assessing barriers to their use. Canadian Medical Association Journal. 2010;182(2):E78-84.

3. Gabbay J, le May A. Evidence based guidelines or collectively constructed "mindlines?" Ethnographic study of knowledge management in primary care. BMJ. 2004;329(7473):1013.

4. Twaddle S. Clinical practice guidelines. Singapore Medical Journal. 2005; 46(12):681.

5. Bierbaum M, Braithwaite J, Arnolda G, Delaney GP, Liauw W, Kefford R, et al. Clinicians' attitudes to oncology clinical practice guidelines and the barriers and facilitators to adherence: a mixed methods study protocol. BMJ open. 2020;0:e035448.

6. Broom A, Adams J, Tovey P. Evidence-based healthcare in practice: a study of clinician resistance, professional de-skilling, and inter-specialty differentiation in oncology. Social Science \& Medicine. 2009;68(1):192-200.

7. Cabana M, Rand C, Powe N, Wu A, Wilson M, Abboud P-A, et al. Why don't physicians follow clinical practice guidelines?: A framework for improvement. JAMA. 1999;282(15):1458-65.

8. Grimshaw J, Thomas R, MacLennan G, Fraser C, Ramsay C, Vale L, et al. Effectiveness and efficiency of guideline dissemination and implementation strategies. Health Technology Assessment. 2004;8(6).

9. Eccles M, Grimshaw J, Walker A, Johnston M, Pitts N. Changing the behavior of healthcare professionals: the use of theory in promoting the uptake of research findings. Journal of Clinical Epidemiology. 2005;58(2):107-12.

10. Damschroder L, Aron D, Keith R, Kirsh S, Alexander J, Lowery J. Fostering implementation of health services research findings into practice: a consolidated framework for advancing implementation science. Implementation Science. 2009;4(1):50.

11. Francke A, Smit M, de Veer A, Mistiaen P. Factors influencing the implementation of clinical guidelines for health care professionals: a 
systematic meta-review. BMC Medical Informatics and Decision Making. 2008;8(1):38.

12. Fischer F, Lange K, Klose K, Greiner W, Kraemer A, editors. Barriers and strategies in quideline implementation —a scoping review. Healthcare. Multidisciplinary Digital Publishing Institute. 2016;4(3):36.

13. Farquhar C, Kofa E, Slutsky J. Clinicians' attitudes to clinical practice guidelines: a systematic review. The Medical Journal of Australia. 2002; 177(9):502-6.

14. Ploeg J, Davies B, Edwards N, Gifford W, Miller PE. Factors influencing bestpractice guideline implementation: Lessons learned from administrators, nursing staff, and project leaders. Worldviews on Evidence-Based Nursing. 2007:4(4):210-9.

15. Graham ID, Brouwers M, Davies C, Tetroe J. Ontario doctors' attitudes toward and use of clinical practice guidelines in oncology. Journal of Evaluation in Clinical Practice. 2007;13(4):607-15.

16. Heneghan C, Mahtani K, Goldacre B, Godlee F, Macdonald H, Jarvies D. Evidence based medicine manifesto for better healthcare: a response to systematic bias, wastage, error and fraud in research underpinning patient care. Royal Society of Medicine; 2017.

17. Saini P, Loke Y, Gamble C, Altman D, Williamson P, Kirkham J. Selective reporting bias of harm outcomes within studies: findings from a cohort of systematic reviews. BMJ. 2014;349:6501.

18. Song F, Parekh S, Hooper L, Loke Y, Ryder J, Sutton A, et al. Dissemination and publication of research findings: an updated review of related biases. Health Technology Assessment. 2010;14(8):1-193.

19. Mitchell AP, Harrison MR, George DJ, Abernethy AP, Walker MS, Hirsch BR. Clinical trial subjects compared to "real world" patients: Generalizability of renal cell carcinoma trials. American Society of Clinical Oncology; 2014

20. Johnson D. Clinicians' attitudes to clinical practice guidelines. The Medical Journal of Australia. 2003;178(7):354-5.

21. Sinuff T, Muscedere J, Cook DJ, Dodek PM, Anderson W, Keenan SP, et al. Implementation of clinical practice guidelines for ventilator-associated pneumonia: a multicenter prospective study. Critical Care Medicine. 2013; 41(1):15-23.

22. Lugtenberg M, Zegers-van Schaick J, Westert G, Burgers J. Why don't physicians adhere to guideline recommendations in practice? An analysis of barriers among Dutch general practitioners. Implementation Science. 2009; 4(1):54.

23. Fang P, He W, Gomez D, Hoffman K, Smith B, Giordano S, et al. Influence of age on guideline-concordant cancer care for elderly patients in the United States. International Journal of Radiation Oncology Biology Physics. 2017; 98(4):748-57.

24. Chagpar R, Xing Y, Chiang Y-J, Feig B, Chang G, You YN, et al. Adherence to stage-spessscific treatment guidelines for patients with colon cancer. Journal of Clinical Oncology. 2012;30(9):972.

25. Ebner F, Hancke K, Blettner M, Schwentner L, Wöckel A, Kreienberg R, et al. Aggressive intrinsic subtypes in breast cancer: a predictor of guideline adherence in older patients with breast cancer? Clinical Breast Cancer. 2015; 15(4):e189-e95.

26. Davis D, Taylor-Vaisey A. Translating guidelines into practice: a systematic review of theoretic concepts, practical experience and research evidence in the adoption of clinical practice guidelines. Canadian Medical Association Journal. 1997;157(4):408-16.

27. Taba P, Rosenthal M, Habicht J, Tarien H, Mathiesen M, Hill S, et al. Barriers and facilitators to the implementation of clinical practice guidelines: a crosssectional survey among physicians in Estonia. BMC Health Services Research. 2012;12(1):455.

28. Munteanu M. Understanding the Most Frequently Experienced Barriers to the Adherence of Lymphoma Clinical Practice Guidelines (CPGs) and Their Effect on the Lymphoma Physicians' Utilization of CPGs: An Explanatory Mixed-Methods Study (Doctoral dissertation, Drexel University). 2018.

29. Grilli R, Penna A, Zola P, Liberati A. Physicians' view of practice guidelines. A survey of Italian physicians. Social Science \& Medicine. 1996;43(8):1283-7.

30. Graham I, Evans W, Logan D, O'Connor A, Palda V, McAuley L, et al. Canadian oncologists and clinical practice guidelines: a national survey of attitudes and reported use. Oncology. 2000;59(4):283-90.

31. Jorgensen M, Young J, Dobbins T, Solomon M. Does patient age still affect receipt of adjuvant therapy for colorectal cancer in New South Wales, Australia? Journal of Geriatric Oncology. 2014;5(3):323-30.
32. Duggan K, Descallar J, Vinod S. Application of guideline recommended treatment in routine clinical practice: a population-based study of stage IIIIB non-small cell lung cancer. Clinical Oncology. 2016;28(10):639-47.

33. Adelson P, Fusco K, Karapetis C, Wattchow D, Joshi R, Price T, et al. Use of guideline-recommended adjuvant therapies and survival outcomes for people with colorectal cancer at tertiary referral hospitals in South Australia. Journal of Evaluation in Clinical Practice. 2018;24(1):135-44.

34. Landercasper J, Dietrich L, Johnson J. A breast center review of compliance with National Comprehensive Cancer Network Breast Cancer guidelines. The American Journal of Surgery. 2006;192(4):525-7.

35. Rhoads K, Ngo J, Ma Y, Huang L, Welton M, Dudley R. Do hospitals that serve a high percentage of Medicaid patients perform well on evidencebased guidelines for colon cancer care? Journal of Health Care for the Poor and Underserved. 2013;24(3):1180-93.

36. Simons P, Houben R, Backes H, Pijls R, Groothuis S. Compliance to technical guidelines for radiotherapy treatment in relation to patient safety. International Journal for Quality in Health Care. 2010;22(3):187-93.

37. Rayson D, Urquhart R, Cox M, Grunfeld E, Porter G. Adherence to clinical practice guidelines for adjuvant chemotherapy for colorectal cancer in a Canadian province: a population-based analysis. Journal of Oncology Practice. 2012:8(4):253-9.

38. Batumalai V, Shafiq J, Gabriel G, Hanna TP, Delaney GP, Barton M. Impact of radiotherapy underutilisation measured by survival shortfall, years of potential life lost and disability-adjusted life years lost in New South Wales. Australia. Radiotherapy and Oncology. 2018;129(2):191-5.

39. Rossi C, Vecchiato A, Mastrangelo G, Montesco M, Russano F, Mocellin S, et al. Adherence to treatment guidelines for primary sarcomas affects patient survival: a side study of the European CONnective TIssue CAncer NETwork (CONTICANET). Annals of Oncology. 2013;24(6):1685-91.

40. Perrier L, Buja A, Mastrangelo G, Vecchiato A, Sandonà P, Ducimetière F, et al. Clinicians' adherence versus non adherence to practice guidelines in the management of patients with sarcoma: a cost-effectiveness assessment in two European regions. BMC Health Services Research. 2012;12(1):82.

41. Chiew KL, Chong S, Duggan K, Kaadan N, Vinod S. Assessing guideline adherence and patient outcomes in cervical cancer. Asia-Pacific Journal of Clinical Oncology. 2017:13(5):e373-e80.

42. Bristow R, Chang J, Ziogas A, Anton-Culver H. Adherence to treatment guidelines for ovarian cancer as a measure of quality care. Obstetrics \& Gynecology. 2013;121(6):1226-34.

43. Balasubramanian S, Murrow S, Holt S, Manifold I, Reed M. Audit of compliance to adjuvant chemotherapy and radiotherapy guidelines in breast cancer in a cancer network. The Breast. 2003;12(2):136-41.

44. Shamseer L, Moher D, Clarke M, Ghersi D, Liberati A, Petticrew M, et al. Preferred reporting items for systematic review and meta-analysis protocols (PRISMA-P) 2015: elaboration and explanation. BMJ. 2015;349:97647.

45. Hong QN, Pluye P, Fàbregues S, Bartlett G, Boardman F, Cargo M, et al. Mixed methods appraisal tool (MMAT), version 2018. IC Canadian Intellectual Property Office, Industry Canada. 2018.

46. Pace R, Pluye P, Bartlett G, Macaulay A, Salsberg J, Jagosh J, et al. Testing the reliability and efficiency of the pilot Mixed Methods Appraisal Tool (MMAT) for systematic mixed studies review. International Journal of Nursing Studies. 2012;49(1):47-53.

47. QSR International Pty Ltd. NVivo qualitative data analysis software. Version 12. 12 ed 2018.

48. Dixon-Woods M, Agarwal S, Jones D, Young B, Sutton A. Synthesising qualitative and quantitative evidence: a review of possible methods. Journal of Health Services Research \& Policy. 2005;10(1):45-53.

49. Thomas J, Harden A. Methods for the thematic synthesis of qualitative research in systematic reviews. BMC Medical Research Methodology. 2008; $8(1): 45$.

50. Saldaña J. The coding manual for qualitative researchers: Sage; 2015.

51. Byrt T, Bishop J, Carlin J. Bias, prevalence and kappa. Journal of Clinical Epidemiology. 1993;46(5):423-9.

52. Moher D, Liberati A, Tetzlaff J, Altman D. The PRISMA Group. Preferred Reporting Items for Systematic Reviews and Meta-Analyses: The PRISMA statement. PLoS Medicine. 2009;6(7):e1000097.

53. Otte I, Salloch S, Reinacher-Schick A, Vollmann J. Treatment recommendations within the leeway of clinical guidelines: A qualitative interview study on oncologists' clinical deliberation. BMC Cancer. 2017;17(1):780.

54. O'Brien M, Tsao M, Cornacchi S, Hodgson N, Reid S, Simunovic M, et al. Effects of a regional guideline for completion axillary lymph node dissection 
in women with breast cancer to reduce variation in surgical practice: A qualitative study of physicians' views. The Breast. 2016;29:126-31.

55. Shelton R, Brotzman L, Crookes D, Robles P, Neugut A. Decision-making under clinical uncertainty: An in-depth examination of provider perspectives on adjuvant chemotherapy for stage II colon cancer. Patient Education and Counseling. 2019;102(2):284-90.

56. Brouwers M, Makarski J, Garcia K, Akram S, Darling G, Ellis P, et al. A mixed methods approach to understand variation in lung cancer practice and the role of guidelines. Implementation Science. 2014;9(1):36.

57. Bristow B, Aldehaim M, Bonin K, Lam CCK, Wan S, Cao X, et al. Patterns of care related to post-operative radiotherapy for patients with prostate cancer among Canadian Radiation Oncologists and Urologists. Journal of Cancer Education. 2018;33(6):1195-200.

58. Brown B, Young J, Kneebone A, Brooks A, Dominello A, Haines M. Knowledge, attitudes and beliefs towards management of men with locally advanced prostate cancer following radical prostatectomy: an Australian survey of urologists. BJU International. 2016;117:35-44.

59. Carrick S, Redman S, Webster F, Bonevski B, Sanson-Fisher R, Simpson J. Surgeons' opinions about the NHMRC clinical practice guidelines for the management of early breast cancer. Medical Journal of Australia. 1998, 169(6):300-5.

60. Fonteyne V, Rammant E, Ost P, Lievens Y, De Troyer B, Rottey S, et al. Evaluating the Current Place of Radiotherapy as Treatment Option for Patients With Muscle Invasive Bladder Cancer in Belgium. Clinical Genitourinary Cancer. 2018;16(6):e1159-e69.

61. Gattellari M, Ward J, Solomon M. Implementing guidelines about colorectal cancer: a national survey of target groups. ANZ Journal of Surgery. 2001; 71(3):147-53.

62. Grilli R, Apolone G, Marsoni S, Nicolucci A, Zola P, Liberati A. The impact of patient management guidelines on the care of breast, colorectal, and ovarian cancer patients in Italy. Medical Care. 1991;29(1):50-63.

63. Ismaila N, Salako O, Mutiu J, Adebayo O. Oncology guidelines usage in a low-and middle-income country. Journal of Global Oncology. 2018:4:1-6.

64. Jagsi R, Huang G, Griffith K, Zikmund-Fisher BJ, Janz NK, Griggs JJ, et al. Attitudes toward and use of cancer management guidelines in a national sample of medical oncologists and surgeons. JNCCN Journal of the National Comprehensive Cancer Network. 2014;12(2):204-12.

65. Ward JE, Boyages J, Gupta L. Local impact of the NHMRC early breast cancer guidelines: Where to from here? Medical Journal of Australia. 1997: 167(7):362-5.

66. White V, Pruden M, Kitchen P, Villanueva E, Erbas B. The impact of publication of Australian treatment recommendations for DCIS on clinical practice: a population-based,"before-after" study. European Journal of Surgical Oncology (EJSO). 2010;36(10):949-56.

67. Lohr KN, Field MJ. Guidelines for clinical practice: from development to use: National Academies Press; 1992.

68. Vigna-Taglianti F, Vineis P, Liberati A, Faggiano F. Quality of systematic reviews used in guidelines for oncology practice. Annals of Oncology. 2006; 17(4):691-701.

69. Shaneyfelt TM, Mayo-Smith MF, Rothwangl J. Are guidelines following guidelines?: The methodological quality of clinical practice guidelines in the peer-reviewed medical literature. JAMA. 1999;281(20):1900-5.

70. Blonde L, Khunti K, Harris SB, Meizinger C, Skolnik NS. Interpretation and impact of real-world clinical data for the practicing clinician. Advances in Therapy. 2018;35(11):1763-74

71. Hains I, Fuller J, Ward R, Pearson S. Standardizing care in medical oncology: Are web-based systems the answer? Cancer. 2009;115(23):5579-88.

72. Rapport F, Clay-Williams R, Churruca K, Shih P, Hogden A, Braithwaite J. The struggle of translating science into action: foundational concepts of implementation science. J Evaluation Clin Practice. 2018;24(1):117-26.

73. Luxford K, Hill D, Bell R. Promoting the implementation of best-practice guidelines using a matrix tool. Disease Management \& Health Outcomes. 2006;14(2):85-90.

\section{Publisher's Note}

Springer Nature remains neutral with regard to jurisdictional claims in published maps and institutional affiliations.

\section{Ready to submit your research? Choose BMC and benefit from:}

- fast, convenient online submission

- thorough peer review by experienced researchers in your field

- rapid publication on acceptance

- support for research data, including large and complex data types

- gold Open Access which fosters wider collaboration and increased citations

- maximum visibility for your research: over $100 \mathrm{M}$ website views per year

At BMC, research is always in progress.

Learn more biomedcentral.com/submissions 